\title{
Femtocells: Smart Approach Analysis to Macrocells
}

\author{
${ }^{1} R$. Seetharaman ${ }^{2} R$. Vijayaragavan \\ 1 Department of ECE,College of Engineering Guindy,Anna University, Chennai, India. \\ Department of ECE,National Institute of Technology Tiruchirappalli, India.
}

\begin{abstract}
Femtocells are intelligent cellular access points that support any mobile device using standard cellular air interfaces, such as UMTS, CDMA2000 and LTE. They contain cellular radios that are tightly integrated with the existing macrocell radio network and thus create a seamless experience for mobile users as they move in and out of femtocell coverage, whether on an active call or in standby. Femtocells learn their radio frequency $(\mathrm{RF})$ surroundings and use this information to self-optimize their operation. In particular, femtocells use their RF awareness to control interference between themselves and between femtocells and macrocells. The surest way to increase the system capacity of a wireless link is by getting the transmitter and receiver closer to each other, which creates the dual benefits of higher quality links and more spatial reuse. In a network with nomadic users, this inevitably involves deploying more infrastructure, typically in the form of microcells, hotspots, distributed antennas, or relays In this paper contains the technical view for femtocells, and describe the state-of-the-art on each front. Also describe the technical challenges facing femtocell networks, and give some preliminary ideas for how to overcome them.
\end{abstract}

\section{Keywords}

LTE-Advanced, 3GPP, Relay deployment, backhaul, amplify and forward relaying.

\section{INTRODUCTION}

The demand for higher data rates in wireless networks is unrelenting, and has triggered the design and development of new data-minded cellular standards such as WiMAX (802.16e), 3GPP's High Speed Packet Access (HSPA) and LTE standards, and 3GPP2's EVDO and UMB standards. In parallel, Wi-Fi mesh networks also are being developed to provide nomadic high-rate data services in a more distributed fashion [1]. Although the Wi-Fi networks will not be able to support the same level of mobility and coverage as the cellular standards, to be competitive for home and office use, cellular data systems will need to provide service roughly comparable to that offered by Wi-Fi networks[2]. Femtocells are aimed at providing high performance $3 \mathrm{G}$ voice and data communications in and around the immediate home environment. Connected to the Operator's mobile network over existing broadband connections in the home, femtocells have the potential to make indoor coverage for mobile communications truly pervasive while delivering additional benefits to both the Operator and end-user[3]. The femtocell network architecture provides Operators with a complete indoor coverage solution, all in a small, low-cost, low-power, easy-to-install base station that can be seamlessly integrated into existing mobile networks and provisioned for service within minutes of switching it on[4].

The main problem to this continued of cellular networks is that the network infrastructure for doing so is expensive. A recent development are femtocells, also called home basestations[5],[6],[7], which are short range, low cost and low power base-stations, installed by the consumer for better indoor voice and data reception. The user-installed device communicates with the cellular network over a broadband connection such as DSL, cable modem, or a separate RF backhaul channel[8]. While conventional approaches require dual-mode handsets to deliver both in-home and mobile services, an in-home femtocell deployment promises fixed mobile convergence with existing handsets. Compared to other techniques for increasing system capacity, such as distributed antenna systems [9] and microcells [10], the key advantage of femtocells is that there is very little upfront cost to the service provider. Voice networks are engineered to tolerate low signal quality, since the required data rate for voice signals is very low, on the order of $10 \mathrm{kbps}$ or less[11]. Data networks, on the other hand, require much higher signal quality in order to provide the multi- Mbps [12]data rates users have come to expect. For indoor devices, particularly at the higher carrier frequencies likely to be deployed in many wireless broadband systems, attenuation losses will make high signal quality and hence high data rates very difficult to achieve[13]. The subscriber is happy with the higher data rates and reliability; the operator reduces the amount on traffic on their expensive macrocell network, and can focus its resources on truly mobile users[14]. To the key arguments in favor of femtocells are the following. Better coverage and capacity. Due to their short transmit-receive distance, femtocells can greatly lower transmit power, prolong handset battery life, and achieve a higher signal-to- interference-plusnoise ratio (SINR). These translate into improved reception the so-called five-bar coverage and higher capacity. Because of the reduced interference[15], more users can be packed into a given area in the same region of spectrum, thus increasing the area spectral efficiency [16],[17], or equivalently, the total number of active users per $\mathrm{Hz}$ per unit area. Improved macrocell reliability. If the traffic originating indoors can be absorbed into the femtocell networks over the IP backbone[18], the macrocell BS can redirect its resources towards providing better reception for mobile users[19].

To summarize, the capacity benefits of femtocells are attributed to:

1. Reduced distance between the femtocell and the user, which leads to a higher received signal strength 
2. Lowered transmit power, and mitigation of interference from neighboring macrocell and femtocell users due to outdoor propagation and penetration losses.

3. As femtocells serve only around 1-4 users, they can devote a larger portion of their resources (transmit power \& bandwidth) to each subscriber. A macrocell, on the other hand, has a larger coverage area $(500 \mathrm{~m}-1 \mathrm{~km}$ radius), and a larger number of users; providing Quality of Service (QoS) for data users is more difficult.

\subsection{Technical Challenges}

The key technical challenges facing femtocell networks.

1. Broadband Femtocells: Resource allocation, timing/synchronization and backhaul.

2. Voice Femtocells: Interference management in femtocells, allowing access to femtocells, handoffs, mobility and providing Emergency-911 services.

3. Network Infrastructure: Securely bridging the femtocell with the operator network over IP.

\section{LTE FEMTOCELLS}

Femtocells operate well with CDMA technology, they will also be able to be used with LTE, long term evolution systems. LTE uses OFDM as the signal format, and therefore LTE femtocells will require development to be undertaken to ensure that the optimum operation is achieved[20]. Femtocells are now an integral part of the development strategy for cellular telecommunications operators[21]. Accordingly the use of femtocells will become a mainstay in the cellular telecommunications roadmap for the future.

\subsection{Femtocell Considerations and \\ Characteristics}

Femtocells overcome the issue of providing effective indoor coverage from the $3 \mathrm{G}$ macro layer by their placement in the end-users' homes. Once installed in an end-user's home a femtocell will enable the Operator to provide higher-quality and higher-performance wireless voice and $3 \mathrm{G}$ data services in and around the immediate vicinity of the home environment[22]. Femtocells are in many ways similar to Wi-Fi access points in that they enable access through an unobtrusive device; however femtocells enable full $3 \mathrm{G}$ service delivery in the home. Similar in size to a DSL gateway or cable modem[23], a femtocell is a low capacity base-station, radiating only sufficient power to cover the area of a home environment. The femtocell connects to the Operator's core network using open 3GPP based standards through the end-user's household broadband internet connection rather than traditional cellular backhaul methods[24].

Accordingly femtocells must also fulfill a number of other criteria:

Low-impact - Space may be limited for some households. As a result femtocells must be physically small, ideally aesthetically pleasing and easy to position. Furthermore, they should also be silent in operation, generate low levels of heat output and inexpensive to run in terms of on-going costs.

Low RF power - The transmit RF power output of femtocells is low; between 10 and 100 milli-watts. Put in perspective, this is a lower power level than many Wi-Fi access points, which can be specified up to 1 Watt of output power. Additionally, by being close to the femtocell the $3 \mathrm{G}$ handset is itself able to transmit at lower power levels than it might otherwise have to when on the macro network.

Backhaul via broadband- Femtocells utilize Internet protocol (IP) and flat base-station architectures. Backhaul connection to Operator networks will be through wired broadband Internet service existing in the home such as DSL, cable, or fibre optics as available[25]. There are no connections required to the wider cellular network other than through the IP core. This will benefit Operators by effectively offloading traffic that would otherwise be on the macro-layer directly onto the internet from the femtocell, this not only reduces the load on the core network, but also lowers the cost of delivering wireless traffic when compared to the macro network.

\subsection{MIMO Femtocells}

Multiple antennas at the transmitter and/or the receiver (MIMO) exploit the spatial diversity of the wireless channel. Femtocells can perform temporal link adaptation through adaptive modulation and coding; additionally, MIMO spatial link adaptation will enable a femtocell to switch between providing high data rates and robust transmission. High data rates are obtainable by transmitting multiple spatial streams (spatial multiplexing) over high SINR links. Over low SINR links, MIMO provides robustness through open and closed loop diversity schemes such as space-time codes and beam forming. Interesting areas for future research are a) link adaptive mode switching for femtocells between diversity and spatial multiplexing [26], b) analyzing the effect of channel state information errors induced by co-channel interference on MIMO femtocell performance[27], c) the complexity limitations of MIMO femtocell receivers, which may be significant vs. macrocell receivers due to cost considerations, and d) channel models for MIMO femtocells, since the diversity characteristics may be very different from macrocells[28]. Femtocells are small cellular telecommunications base stations that can be installed in residential or business environments either as single standalone items or in clusters to provide improved cellular coverage within a building. By using a small internal base station - femtocell, cellular coverage, especially for data transmission where good signal strengths and cellular performance can be improved. In order to link the femtocells with the main core network, the mobile backhaul scheme uses the user's DSL or other Internet link. There are many advantages for the deployment of femtocells to both the user and the mobile network operator[29]. For the user, the use of a femto cell within the home enables far better coverage along with the possible provision of additional services.

\section{FEMTOCELL RADIO ACCESS TECHNOLOGY}

The tiny cells created by femtocells typically lie inside larger cells served by nearby macrocell base stations. To operate such an underlay network reliably, femtocells need to avoid or strongly mitigate any interference with macrocells and provide a seamless experience to users as they roam in and out of femtocell coverage. Since existing macrocell networks and mobile devices have been designed without awareness of femtocells, these requirements must be met without requiring any changes to mobile devices. The underlying femtocell radio technologies used in CDMA and UMTS femtocells are quite similar. An important difference between these two systems to note at the outset is that in UMTS[30], voice and high-speed data (HSxPA) services can be delivered on the same $5 \mathrm{MHz}$ wide radio channel, whereas in CDMA these services are delivered on different $1.25 \mathrm{MHz}$ wide radio channels using two different air interfaces: CDMA2000 1x is used for voice services and CDMA2000 EV-DO is used for broadband data. Interference Avoidance through Frequency Planning a mobile operator has three basic options for allocating available frequencies in femtocell deployments. 
These are represented by the scenarios A, B and C in Figure 1, below.

Scenario A represents a dedicated radio channel femtocell deployment that provides separate macrocell and femtocell radio channels. This has the advantage of minimizing interference between the two networks and simplifies initial deployment of femtocells. Scenario A is typically more suitable in rural areas where the mobile operator may have unused radio channels. Scenario A is also more feasible in CDMA2000 systems where radio channels are more numerous because they have a narrower bandwidth. Scenario $\mathrm{C}$ shares all available radio channels between the macrocell and femtocell networks. This has the advantage of providing more degrees of freedom to manage interference between femtocells, especially in dense urban deployments, but also requires the greatest degree of interference management to ensure minimal impact on the macrocell network from the cochannel femtocells. Scenario B represents a compromise between scenarios $\mathrm{A}$ and $\mathrm{C}$ in which some radio channels are shared between the macrocell and femtocell networks and other radio channels are reserved for the macrocell network only. In Scenario B the macrocell can redirect the mobile devices it is serving on the shared radio channel to a dedicated macrocell radio channel when they approach a femtocell. One way of deploying scenario $B$ is to use the shared radio channel primarily for macrocell data services (HSxPA), and for femtocell voice and data services, and leave the dedicated radio channels for macrocell voice services. Since mobile operators may not be able to dedicate radio channels to femtocells in many of their markets, femtocells need to be designed with advanced interference mitigation techniques that allow reliable operation when femtocell and macrocell networks share the same radio channels - Scenario C.

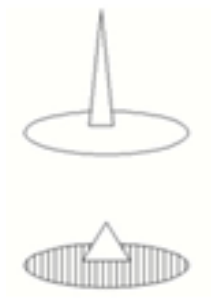

(a)

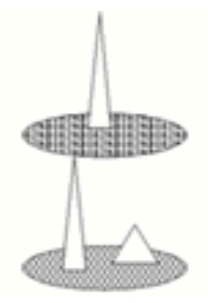

(b)

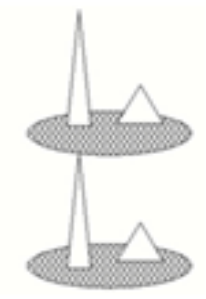

(c) (a) Dedicated Carrier

(b) One Shared Carrier and One macro-only Carrier

(c) All Carriers shared between femtocells and marocells

\section{Figure 1. Deployment Scenarios for Sharing Radio Channels Between Macrocell and Femtocell networks.}

Femtocell Networks Interference Mitigation in Co-Channel Femtocell Deployments Sharing all available spectrum between femtocell and macrocell networks ultimately leads to the most efficient utilization of available spectrum, provided proper interference mitigation techniques are employed. In the discussion of interference mitigation techniques below, we consider separately interference on the Downlink (DL) or equivalently Forward Link (FL) from the base station to the mobile device and interference on the Uplink (UL) or equivalently Reverse Link (RL) from the mobile device towards the base station. Also, we consider separately interference affecting users of the femtocell and users of macrocell. Forward Link/Downlink Interference Mitigation A femtocell must set its FL/DL transmission power high enough to overcome the interfering macrocell signal within the femtocell's target coverage area. But the femtocell cannot arbitrarily increase its transmission power, as this would generate interference to mobile devices nearby that are operating on the same radio channel but are being served by a macrocell base station or another femtocell. To deal with this conundrum femtocells set their transmission power adaptively. They measure the strength of the signals received from nearby macrocells and other femtocells and set the FL/DL transmission power level just high enough to achieve acceptable SNR inside the target coverage area. Femtocells also obtain measurement reports from nearby mobile devices, and track the presence of mobile devices being served by macrocell base stations to fine tune the FL/DL transmission power level and deliver the best possible grade of service to all femtocell and macrocell users nearby. A femtocell transmitting at too high a power level creates interference to a nearby mobile device that is being served on the same radio channel by a far away macrocell. This can create a "dead zone" where even basic voice communication with the macrocell base station may become impossible. Femtocells avoid this scenario by either pushing these mobile devices away to another radio channel on the macrocell network while they are still in standby mode or by allowing them to park on the femtocell in standby mode, and handing them out to a different radio channel on the macrocell network whenever they turn active for a voice or data call[31]. This way all macrocell calls in the close vicinity of the femtocell always take place on a different radio channel and thus avoid any interference from the femtocell. In the scenario where a mobile device starts a call with the macrocell outside the femtocell coverage area and moves close to the femtocell while still active on the call, the call quality can degrade as it approaches the femtocell. Upon detecting the strong pilot signal of the femtocell, the mobile device will report to its macrocell base station that the SNR it is experiencing has decreased and that another cell with a stronger signal is nearby. A well-designed macrocell radio controller can recognize that the interfering cell is a femtocell, and redirect the mobile device to another radio channel, i.e., perform interfrequency handoff, thus avoiding any interference that may be caused by the femtocell.

\subsection{Reverse Link/Uplink Interference Mitigation}

Macrocell base stations maintain system stability on the RL/UL by controlling the total received RL/UL power. The transmission power of mobile devices that are being served by the macrocell base station are controlled in such a way that the rise in total received power over the equivalent thermal (ambient) noise level is maintained at or below a predetermined threshold. This threshold, also known as the RiseOver-Thermal (RoT), is typically set at between 5 and $10 \mathrm{~dB}$. Power control equalizes the strength of signals being received from mobile devices that are at different distances from the base station, and thereby maintains system stability. Soft handoff procedures also allow multiple base stations to control the transmission power of the mobile device located at a cell boundary. Mobile devices that are being served by macrocells will set their transmission power in a way that is oblivious to the presence of femtocells. Because the distance between a mobile device and a macrocell is typically much larger than that between the device and a nearby femtocell, the RL/UL signal received by the femtocell from such a device 
can be very high, raising the interference level up to 30 or 40 $\mathrm{dB}$ above levels typically seen in macrocell base station receivers. The femtocell receiver hardware is designed to handle such high levels of interference from nearby mobile devices, without suffering from any saturation effects. The femtocell will instruct the mobile devices it is serving to raise their transmission power to overcome the interference from nearby mobile devices being served by a macrocell base station, using a variation of the power control algorithm used in macrocell base stations. Having to raise its transmission power poses no problem for the mobile device being served by the femtocell because its transmitter is designed for operation with distant macrocell base stations. It generally has plenty of power available to reliably communicate with a nearby femtocell, even in presence of interference from other nearby mobile devices transmitting to a macrocell at a high power. In fact, in the absence of any interference from other mobile devices, a mobile device being served by a femtocell uses very little transmission power relative to what it uses on a macrocell network, which leads to longer battery life (or more specifically, talk time) and avoids RL/UL interference to nearby macrocell base stations[32].

However in the scenario where a mobile device being served by femtocell has to raise its transmission power in response to interference being caused by a mobile device being served by a faraway macrocell base station, elevated interference levels can occur in the macrocell base station. As macrocell base stations are designed to operate in a power-controlled environment, unplanned interference from such a mobile device can cause mobile users being served by the macrocell near the cell edge to experience lower data throughput and call drops. Femtocells avoid this phenomenon by constantly evaluating the interference its mobile devices are causing to nearby macrocell base stations, and ensure that such interference does not reach levels where they affect macrocell user experience. This is done by using measurement reports from mobile devices to evaluate their path loss to the nearest macrocell base station, and by limiting their transmission power using power control algorithms.

\section{RESEARCH METHODOLOGY APPROACH}

\subsection{LTE Femtocell Architecture}

The concept behind the LTE SAE is to provide a much flatter overall network architecture. This has many advantages in terms of network simplification and it is also a key element in enabling much lower levels of latency - a key requirement for LTE.

The femtocell network architecture has been defined to allow maximum flexibility and scalability to ensure that the deployment can be easily incorporated into the existing structures. By its very nature, the deployment of femtocells is achieved on an ad-hoc basis; this forms a large requirement for the system.

\subsection{Synchronization}

3GPP specifies that base station frequencies need to be very accurate, and there needs to be close synchronisation with precise clock signals. Reasons for femtocell synchronization, Supply frequency information to handsets, Ensure reliable handover, Interference reduction, Ensures femtocell to be aware of adjacent cell sites[33]. If the femtocell is accurately synchronized to the rest of the network it can detect other cells more quickly and thereby improve the operation of the femtocell.

\subsection{Levels of accuracy}

Accuracy levels are defined in many standards for different types of cell. These tend to vary according to the type of cell which the base station is serving.

\begin{tabular}{|l|c|}
\hline BASE STATION TYPE & $\begin{array}{c}\text { FREQUENCY } \\
\text { ACCURACY (PPB) }\end{array}$ \\
\hline Wide area & 50 \\
\hline Local area & 100 \\
\hline Home-femtocell & 250 \\
\hline
\end{tabular}

Table 1: Base Station Types (PPB) Comparison

It would be possible to utilise a very high stability standard to produce the required level of accuracy and hence femtocell synchronization. However cost can be an issue and therefore other methods of obtaining the required level of femtocell synchronization and frequency accuracy may be more effective.

\subsection{Handover}

Femtocell handover techniques need to ensure that seamless coverage is perceived by the user when moving onto or off a femtocell. Femtocell handover is more challenging than normal macrocell cellular handover because the backhaul network is different and there is also little possibility of direct communication between the femtocell and the macrocell. There are a number of scenarios for femtocell handover:

Inbound: This is where handover occurs from the macro-cell or standard cellular network to the femtocell. Outbound: This is where a handover occurs from the femtocell to the macro-cell or standard cellular network. Femtocell to femtocell: There will be situations where handover will occur between one femtocell and another close by. This will be commonplace in offices that may have a number of femtocells to give continuous coverage within a building.

\subsection{Femtocell security risks \& Health issues}

There are a number of concerns that exist about femtocell security. By categorizing these femtocells security concerns it is possible to address them and ensure that any risks are minimized. User privacy, Denial of service and general service availability, Fraud and service theft. One of the concerns that could be a concern with the use of femtocells is that of the resultant health issues associated with the use of radio frequency radiation. The standards for radio frequency radiation are set to levels that allow radio transmissions of various forms to be used, but at levels where current scientific research and thinking indicates that there are no unreasonable risks.

\section{NETWORK INFRASTRUCTURE}

In a femtocell environment, the operator will need to provide a secure and scalable interface for the femtocell over IP, at a reasonable cost. Traditional Radio Network Controllers (RNCs) are equipped to handle tens to hundreds of macrocells. .Three network interfaces have been proposed, of which the IMS/SIP and UMA based interfaces appear to be the architectures of choice. Iu-b over IP: Existing RNCs connect to femtocells through standard Iu-CS (circuitswitched) and Iu-PS (packet-switched) interfaces present in macrocell networks. The advantage is that the Capex is comparatively low insofar as the operator can leverage existing RNCs. The shortcomings are the lack of scalability, and that the interface is not yet standardized. 


\subsection{IMS/SIP}

The Internet Media Sub-System/Session Initiation Protocol interface provides a core network residing between the femtocell and the operator. The IMS interface converts subscriber traffic into IP packets and employs Voice over IP (VoIP) using the SIP protocol, and coexists with the macrocell network. The main advantages are scalability and rapid standardization. Disadvantages include the Capex for upgrade, and Opex in maintaining two separate core networks for the macrocell and femtocell respectively.

\subsection{RAN gateway based UMA}

A Radio Access Network (RAN) gateway exists between the IP network and the operator network, aggregating traffic from femtocells. This gateway is connected to the operator network using a standard Iu-PS/CS interface. Between the femtocell and the RAN gateway, the UMA (Unlicensed Mobile Access) protocol makes use of secure IP tunneling for transporting the femtocell signals over the internet.

\subsection{Interference Management}

Owing to the ad-hoc topology of femtocell locations, interference suppression techniques alone will prove ineffective in femtocell networks. Successive Interference Cancellation - in which each user subtracts out the strongest neighboring interferers from their received signal-appears promising initially, but cancellation errors quickly degrade its usefulness [12]. Consequently, an interference avoidance approach wherein users avoid rather than suppress mutual interference is more likely to work well in geographydependent femtocell networks. The low cost requirement is likely to influence the design of low complexity femtocell BS receivers - simple matched filter processing for exampleand low complexity transmission schemes for sensing nearby available frequency channels to avoid collisions.

In CDMA femtocell networks with universal frequency reuse, for example, interference avoidance-through time-hopping and directional antennas - provides a $7 \mathrm{x}$ improvement in system capacity [34], when macrocell and femtocell users share a common bandwidth.

\subsection{Frequency-and Time-hopping}

In GSM networks, the interference avoidance offered through slow frequency hopping enables femtocell users and nearby transmitting macrocell users to avoid consistent mutual interference. Similarly, frequency-hopped OFDMA networks can use random sub-channel assignments in order to decrease the probability of persistent collision with neighboring femtocells.

In time- hopped CDMA, the CDMA duration $\mathrm{G} T(\mathrm{G}$ is the processing gain and $\mathrm{T}$ is the chip period) is divided into $\mathrm{N}$ hopping slots, where each user randomly selects a hopping slot for hop transmission and remains silent during the remaining slots. Random time-hopping reduces the average number of interfering users by a factor of $\mathrm{N}$, while trading-off the processing gain. Hop The tradeoff is that femtocells are accommodated by changing the way an existing CDMA macrocell network operates. Directional Antennas inside femtocells offer interference avoidance, with zero protocol overhead, by restricting radio interference within an antenna sector. Providing a reasonable unit cost and easy end user deployment are the key challenges confronting this approach[35]. Adaptive Power Control strategies vary the femtocell receive power target depending on its location. Commercial femtocells such as Sprint's femtocell tackle cross-tier interference using an "automatic adaptation" protocol that adjusts the femtocell transmit power. Over the forward link in closed access, has proposed reducing interference to macrocell users by reducing the femtocell transmit power with increasing distance from the macro BS. The tradeoff is the decreased home coverage at far-off femtocells. Over the reverse link in closed access, we suggest providing a higher receive power target to femtocell users relative to macrocell users, which will vary based on femtocell location.

\section{NETWORK ARCHITECTURE}

Connecting femtocells to operator networks requires unique architectures that address the security needs of operators and mobile users and support the deployment of scalable femtocell networks that can serve millions of subscribers. Network architecture is also critical in supporting emergency calling, which can now be delivered to mobile devices inside buildings with the same reliability and accuracy as fixed-line emergency calling. Connecting femtocells to existing operator networks requires a network architecture that addresses the security needs of operators and mobile users, while supporting the scalable deployment of millions of femtocells. Some of the key reqirements: 1) Service ParityFemtocells support the same voice and broadband data services that mobile users are currently receiving on the macrocell network[36]. This includes circuit-switched services such as text messaging and various voice features, such as call forwarding, caller ID, voicemail and emergency calling. 2) Call Continuity- Femtocell networks are wellintegrated with the macrocell network so that calls originating on either macrocell or femtocell networks can continue when the user moves into or out of femtocell coverage. Femtocell network architecture needs to include the necessary connectivity between the femtocell and macrocell networks to support such call continuity. 3) Security- Femtocells use the same over-the-air security mechanisms that are used in macrocell radio networks. But additional security capabilities need to be supported to protect against threats that originate from the Internet or through tampering with the femtocell itself. Femtocell network architecture provides network access security, and includes subscriber and femtocell authentication and authorization procedures to protect against fraud. 4) Scalability- Femtocell networks can have millions of access points. Therefore the femtocell network architecture must be scalable to grow into such large networks, while at the same time maintaining reliability and manageability[37]. Common Elements of the Femtocell Network Architecture As shown in Figure 2 there are three network elements that are common to any femtocell network architecture. These are:

- Femtocell Access Point (FAP)

- Security Gateway (SeGW)

- Femtocell Device Management System (FMS)

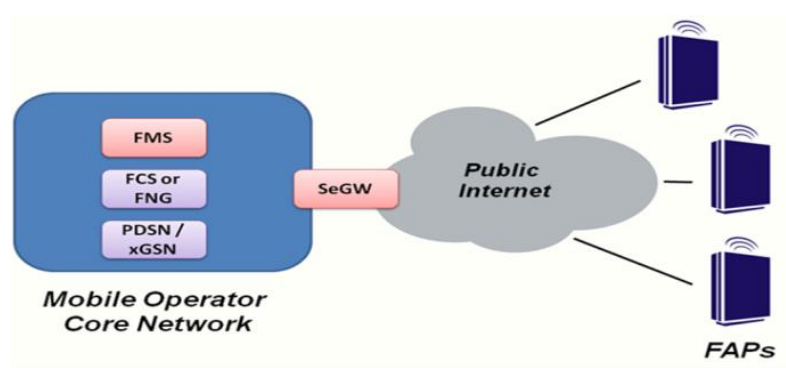

Figure 2: Network Architecture 
Two other elements that are in all femtocell network architectures are entities that enable connectivity to the mobile operator core. Depending on the specific architecture used for circuit switched calls, there can be either a Femtocell Convergence Server (FCS) or a Femtocell Network Gateway (FNG). This is also shown in Figure 2. For packet calls, depending on the airlink technology, there can be either a PDSN or xGSN (GGSN/SGSN) in the core. In most cases, the PDSN / $\mathrm{xGSN}$ are the same as those used for macro networks. Figure 2: Common Components of Femtocell Network Architecture Femtocell Access Point (FAP) Femtocell Access Point is the primary node in a femtocell network that resides in the user premises (e.g., home or office). The FAP implements the functions of the base station and base station controller and connects to the operator network over a secure tunnel via the Internet[38]. A FAP can be introduced into a home in multiple ways. A standalone FAP can be directly connected to the home router. In some applications, the FAP may also include a built-in router, which is useful in prioritizing FAP voice traffic over other Internet traffic in the home network. More advanced FAP's include an Analog Terminal Adapter (ATA) to connect a fixed-line phone. In some cases, FAP's are fullblown residential gateways with built-in Wi-Fi and a broadband modem (xDSL, cable).

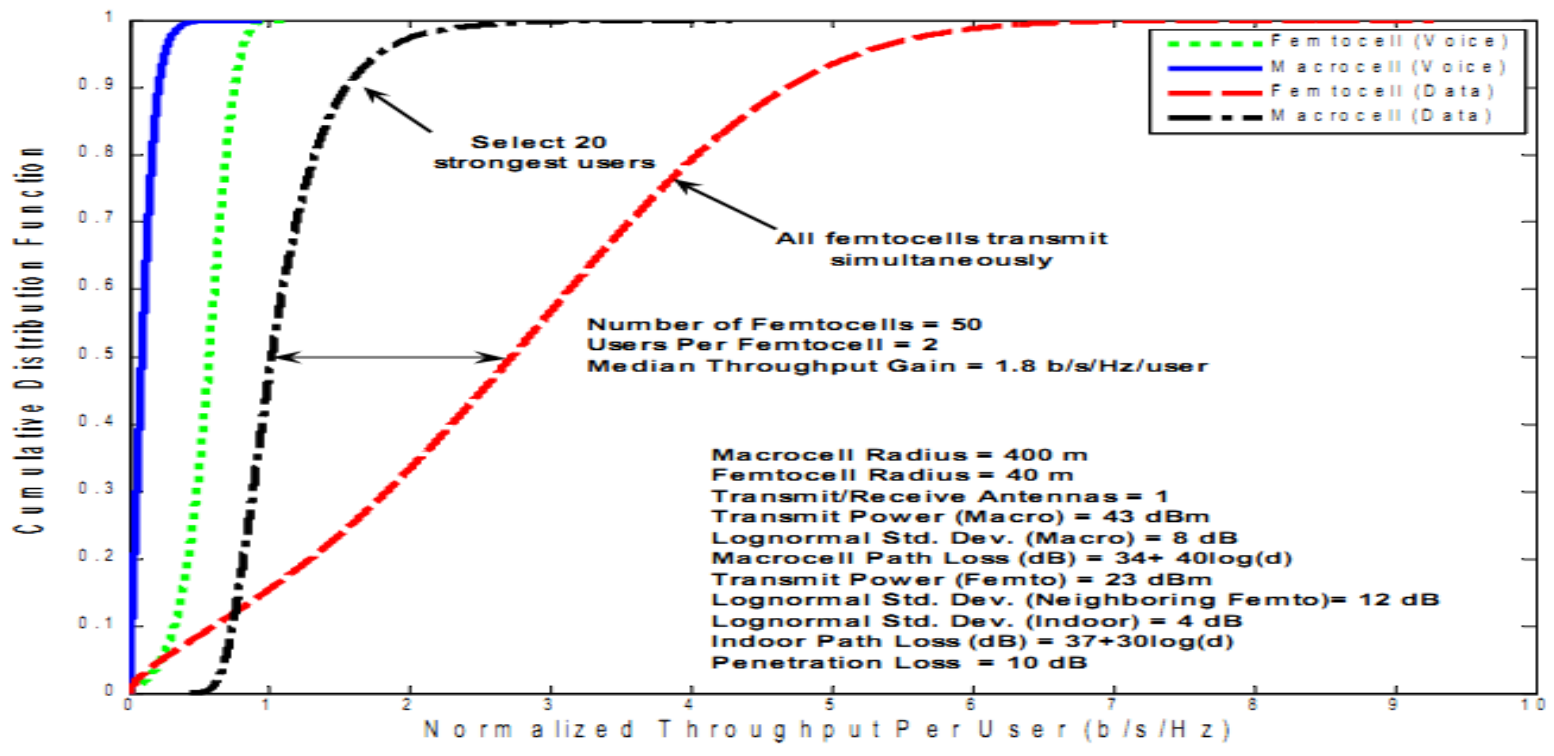

Figure 3: Femtocell Vs Macrocell Throughput

\section{CONCLUSION}

Femtocells are not simple standalone devices. They must be integrated into the mobile operator's network to enable seamless service and to ensure optimal performance across both femtocell and macrocell networks. The architectures for the UMTS and CDMA solutions have been defined by their respective standards bodies (3GPP and 3GPP2). Both architectures enable a better experience, with service parity for users, while ensuring security and scalable solutions for operators. Considering that the majority of "mobile" calls originate in the home and end-users prefer to use a single handset - their mobile, Operators now have focused solutions available to them that overcome the issues of poor in-building coverage. Femtocells will provide a one-box solution: a small, low-cost, low power unit that can be self- installed to provide mobile $3 \mathrm{G}$ coverage to the home. For the end-user femtocell solutions will provide dedicated and reliable mobile $3 \mathrm{G}$ coverage in the home with opportunities for preferential tariffs. research opportunities. From a technical standpoint, operators face challenges in providing a low cost solution, while mitigating RF interference, providing QoS over the IP backhaul, and maintaining scalability. From a business perspective, generating long-term revenue growth and overcoming initial end user subsidies are key challenges.

Furthermore, by adopting a femtocell solution, Operators can easily add $3 \mathrm{G}$ mobile with in-home usage tariffs to a bundled mobile and broadband offering without the macrolayer bearing the Femtocells have the potential to provide high quality network access to indoor users at low cost, while simultaneously reducing the burden on the whole system. This article has identified the key benefits of femtocells, the technological and business challenges. 


\section{REFERENCES}

[1] Saleh.A. Rustako.A and Roman.R. 1987. Distributed Antennas for Indoor Radio Communications. IEEE Transactions on Communications. vol. 35, no. 12, pp. $1245-1251$.

[2] Chih- Lin I. Greenstein.L.j. and Gitlin.R.D. 1993. A Microcell/Macrocell Cellular Architecture for Low- and High-Mobility Wireless Users. IEEE Journal on Selected Areas on Communication, vol. 11 , no. 6 , pp. $885-891$.

[3] Analysys, Picocells and Femtocells: Will indoor base-stations transform the telecoms industr $y$ ? [Online]. Available: http://research.analysys.com

[4] Alouini.M.S. and Goldsmith.A.J. 1999. Area Spectral Efficiency of Cellular Mobile Radio Systems. IEEE Transactions on Vehicular Technology, vol. 48, no. 4, pp. $1047-1066$

[5] Picochip. 2007. The Case For Home Base Stations. White Paper.

[6] Andrews.j.g. Ghosh.A. and Muhamed.R. 2007. Fundamentals of WiMAX. Prentice-Hall.

[7] Chandrasekhar.V. and Andrews.J. Uplink Capacity and Interference Avoidance in Two- Tier Femtocell Networks. to appear, IEEE Trans. On Wireless Communications , Available at http://arxiv.org/abs/cs.NI/0702132.

[8] Lester.T.W. Ho and Holger Claussen. 2007. Effects of User-Deployed, Co-Channel Femtocells on the Call Drop Probability in a Residential Scenario. The $18^{\text {th }}$ Annual IEEE International Symposium on Personal, Indoor and Mobile Communications, pp. $1-5$.

[9] Kwan.L. Yeung and Sanjiv Nanda. 2006. Channel Management in Microcell/Macrocell Cellular Radio Systems. IEEE Trans. On Vehicular Technology, vol. 45, no. 4, pp. $601-612$.

[10] Weber.s. Andrews.j.G. Yang.X. and de Veciana.G. 2007. Transmission Capacity of Ad Hoc Networks with Successive Interference Cancellation. IEEE Trans. on Information Theory, vol. 53, no. 8, pp. 2799 -2814 .

[11] Ericsson. Home NodeB Output Power. 3GPP TSG Working Group 4 meeting, [Online]. Available: http://www.3gpp.org/ftp/tsg_ran/WG4_Radio/TSGR4 _43bis/Docs/

[12] Kishore.S. Greenstein.L.J. Poor.H.V. and Schwartz.S.C. 2006. Uplink User Capacity in a Multicell CDMA System with Hotspot Microcells. IEEE Trans. On Wireless Communications, vol. 5, no. 6, pp. $1333-1342$.

[13]Chandrasekhar.V. Andrews.J. and Gatherer.A. 2008. Femtocell networks: a survey. IEEE Communications Magazine, vol. 46, no. 9, pp. 59-67.
[14] 3GPP. 2010. Further advancements for e-utra physical layer aspects. Tech. Rep. TR.36.814 v2.0.10, 30.

[15] 3g home nodeb study item technical report. 2008. 3GPP, Tech. Rep. TR.25.820 v8.2.0.

[16] Knisely.D. Yoshizawa.T. and Favichia.F. 2009. Standardization of fem- to cells in 3gpp. IEEE Communications Magazine, vol. 47, no. 9, pp. 68-75.

[17] de la Roche.G. Valcarce.A. Lopez-Perez.D. and Zhang.J. 2010. Access control mechanisms for femtocells. IEEE Communications Magazine vol. 48, no. 1, pp. 33-39.

[18] Chandrasekhar.V. Andrew.J. Gatherer.A. 2008. Femtocell networks: a survey. IEEE Communications Magazine, Volume 46.

[19] Chandrasekhar. V. and Andrews. J. 2007. Uplink Capacity \& Interference Avoidance for Two-Tier Networks; Proceedings of IEEE Global Telecommunications Conference. Washington DC, USA; Pages 3322-3326.

[20] Shu-ping Yeh, Talwar S. Seong-choo $\mathrm{n}$ Lee. Heechang Kim. 2008. WiMAX femtocells: a perspective on network architecture, capacity and coverage. IEEE Communications Magazine. Volume 46, Issue 10; Pages 58 - 65.

[21] Schiller J. Mobile Communications . pages 93-164. $2^{\text {nd }}$ Edition. ISBN 0-321-12381-6.

[22] Hasan,S.F. Siddique N.H. Chakraborty,S. 2009. Femtocell vs. WiFi - A survey and comparison of architecture and performance. $1^{\text {st }}$ International Conference on Wireless Communication, Vehicular Technology, Information Theory and Aerospace \& Electronic Systems Technology. Page(s): 916 - 920.

[23] Holma.H. Toskala.A. WCDMA for UMTS. John Wiley \& Sons. 2002. ISBN 978-0470844670. International Journal of Next-Generation Networks (IJNGN) Vol.3, No.1, March 2011.

[24] Femto Foru. ; Interference Management in UMTS Femtocells . 2008. available online at www.femtoforum.org.

[25] Mahmoud.H. and Guvenc.I. 2009. A comparative study of different deployment modes for femtocell networks. in IEEE 20th International Symposium on Personal, Indoor and Mobile Radio Communications. 13-16 September 2009, pp. 1-5.

[26] Arulselvan.N. Ramachandran.V. Kalyanasundaram.S. and Han.G. 2009. Distributed power control mechanisms for hsdpa femtocells. in Vehicular Technology Conference, 2009. VTC Spring 2009. IEEE $69^{\text {th }} \cdot$ pp. $1-5$.

[27] Yavuz.M. Meshkati.F. S.Nanda. Pokhariyal.A. Johnson.N. . Raghothaman and Richardson.A. 2009. Interference management and performance analysis of 
umts/hspa+ femtocells. IEEE Communications Magazine, vol. 47, no. 9, pp. 102-109.

[28] Claussen.H. D.Calin. 2009. Macrocell of loading benefits in joint macro- and femtocell deployments. in Personal, Indoor and Mobile Radio Communications. IEEE 20th International Symposium on , 13-16 2009, pp. 350-354.

[29] Andrews.M. Capdevielle.V. Feki.A. and Gupta.P. 2010. Autonomous spectrum sharing for mixed lte femto and macro cells deployments. pp. 1-5.

[30] Lopez-Perez.D. Ladanyi.A Juttner.A. and Zhang.J. 2009. Ofdma femto- cells: A self-organizing approach for frequency assignment. pp. 2202-2207.

[31] Morita.M. Matsunaga.Y. and Hamabe. K. 2010. Adaptive Power Level Setting of Femtocell Base Stations for Mitigating Interference with Macro cells. in Vehicular Technology Conference Fall (VTC 2010Fall), 2010 IEEE $72^{\text {nd }}$, vol. , no. pp. 1-5.

[32] Guidelines for evaluation of radio interface technologies for imt- advanced. ITU. Tech. Rep. M2135.

[33] Mogensen.P. Na.W. Kovacs.I. Frederiksen.F. Pokhariyal.A. Pedersen.K. Kolding.T. Hugl.K. and Kuusela.M. 2007. Lte capacity compared to the macro/micro cell throughput is observed. In hybrid control Shannon bound. in Vehicular Technology Conference. 2007. VTC2007. pp. 1234-1238.

[34] Fan.Y. Lunden.P. Kuusela.M. and Valkama.M. 2008. Efficient semi- persistent scheduling for VoIP on e-utra downlink. in Vehicular Tech- nology Conference. VTC 2008-Fall, IEEE $68^{\text {th }}, 2008$, pp. 15 .

[35] Haddad.Y. Porrat.D. 2007. Femtocell: Opportunities an $\mathrm{d}$ challenges of the home cellular base station for 3G. Proceedings of IEEE Global Telecommunications Conference. Washington DC, USA, pp. 3317-3321.

[36] Choi.D. Monajemi .P. Kang.S. Villasenor.J. 2008.Dealing with Loud Neighbours: The benefits and Trade-offs of Adaptive Femtocell Access . IEEE Global Telecommunication s Conference.

[37] Chowdhury. M.Z. Ryu W. Rhee E. Jang Y. M. 2009. Handover between femtocell and macrocell for UMTS-based networks; $11^{\text {th }}$ International Conference on Advanced Communication Technology, 2009; Issue date: 15-18. Volume 1, Pages 2 37-241.

[38] Han.K. Choi.Y. Kim.D. Na.M. Choi.S. Han.K. 2009. Optimization of femtocell network configuration under interference constraints; $7^{\text {th }}$ International Symposium on Modeling and Optimization in Mobile, Ad Hoc, and Wireless Networks.Pages 1-7. 